\title{
Edge states of photon pairs in cavity arrays with spatially modulated nonlinearity
}

\author{
Mark Lyubarov $\circledast^{1, *}$ and Alexander Poddubny ${ }^{1,2,3}$ \\ ${ }^{1}$ Physics and Engineering Department, ITMO University, St. Petersburg 197101, Russia \\ ${ }^{2}$ Nonlinear Physics Centre, Australian National University, Canberra ACT 2601, Australia \\ ${ }^{3}$ Ioffe Institute, St. Petersburg 194021, Russia
}

(Received 28 June 2019; published 6 November 2019)

\begin{abstract}
We study theoretically an extended Bose-Hubbard model with the spatially modulated interaction strength, describing a one-dimensional array of tunneling-coupled nonlinear cavities. It is demonstrated that the spatial modulation of the nonlinearity induces bound two-photon edge states. The formation of these edge states has been understood analytically in terms of nonlinear self-localization. Our results show that the number of excitations and the modulation period of the interaction affect the photonic quantum walks in the universal way and both promote the edge states.
\end{abstract}

DOI: 10.1103/PhysRevA.100.053813

\section{INTRODUCTION}

The quantum simulations with arrays of coupled qubits are now rapidly developing with 51-qubit systems based on the cold atom platform being already available [1,2]. The integrated quantum optical platform potentially promises robust scalable quantum simulations on a chip. It is now under active development [3-8], although still catches up with the cold atom and superconducting resonator networks [9]. Hence it is specially instructive to reexamine the conceptual effects of interaction that can be manifested already for a few interacting particles and are potentially easier to implement.

One of the simplest interaction effects is the formation of spatially bound boson pairs (doublons) that can be present for both attractive and repulsive interaction [10]. Qualitatively, these states form because the energy of bound pairs is repelled either below or above the continuum of quasi-independent scattering states and hence the photons become colocalized. The doublon states arisen due to repulsive interaction have been already observed in a cold atom system [11,12]. The one-dimensional two-particle Bose-Hubbard model, describing the formation of doublons, is very useful despite its conceptual simplicity. First, it can be readily emulated even classically by considering a mathematically equivalent twodimensional array of coupled waveguides $[13,14]$ or even equivalent electric circuits [15]. Second, it can be generalized by including the spatial modulation of the nonlinearity, onsite energies, and tunneling coefficients, which significantly enriches the types of two-particle states available in the system. For instance, in Ref. [16] we recently demonstrated that the formation of bound two-particle states is a quite general phenomenon that can occur even when the interaction is dissipative. In Ref. [17] the Tamm-Hubbard two-photon edge states were found in the system, where the edge cavity has been detuned by energy from those in the bulk.

\footnotetext{
*markljubarov@gmail.com
}

Recent interest to the localized biphoton states has been stimulated by the rapid progress of topological photonics that promises disorder-robust edge states of light $[18,19]$. While the topological edge states of classical electromagnetic waves have already been known for a decade [20], the quest for nonlinear and quantum topological photonics systems has begun quite recently $[6-8,21-23]$. The photon pairs in the arrays of coupled cavities allow one to study the interplay of topology and interactions [24]. It has been predicted that the spatial modulation of the tunneling constants in the array of nonlinear cavities can induce the formation of the twophoton edge states [25-28]. The physics of this system is unexpectedly rich. For instance, contrary to the classical SuSchrieffer-Heeger model describing noninteracting particles in the array of cavities with modulated interaction strength [29], the edge states for the pair of interacting photons can arise at the edges with both weak and strong tunneling links [26]. Another interesting mechanism to realize the two-photon edge states is to implement the nonlocal interactions, when the photon energy is modified by the presence of the photon in the adjacent cavity [30].

Here, we study how the interplay of the spatial modulation of the structure and the nonlinearity affects the many-photon edge states. We start the one more extension of the BoseHubbard model by considering the array of cavities where all single-photon energies and tunneling links are the same, but the interaction strength is spatially modulated; see Fig. 1 . We demonstrate that this system also possesses bulk doublon states and edge doublon states. Next, we analyze the graph of two-photon quantum walks and demonstrate that these edge states are an inherent feature of the spatially modulated local on-site nonlinearity. This differs them qualitatively from the topological edge states considered in Refs. [15,26], where the single- and two-photon tunneling amplitudes have been modulated in space. We also consider the three-photon states in the modulated structures, generalizing the consideration of simple periodic structures in Ref. [31]. We demonstrate that the number of excitations and the modulation period of the interaction universally affect the photonic quantum walks. 


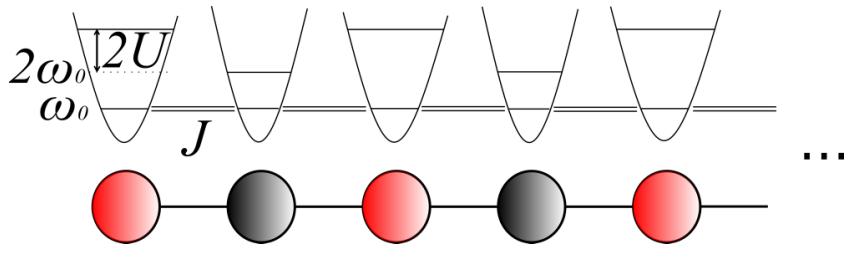

FIG. 1. Schematics of the considered array of qubits with the single-photon resonance frequency $\omega_{0}$. The modulated interaction strength $2 U$ is illustrated by the effective potentials with different anharmonicity.

Namely, both the increase of the excitation number and the modulation period promotes the appearance of many-photon edge states.

Most flexible realization of the proposed model with spatially modulated interactions in the solid-state system could probably be based on the exciton-polaritons, half-light halfmatter quasiparticles [32]. The interaction between polaritons depends on their excitonic fraction and can be modified by controlling the spectral detuning between the exciton and the cavity. However, observation of the interactions at the quantum level of single polariton is an ongoing and challenging quest. First experimental indications of the polariton blockade effect have appeared only very recently [33,34]. At the moment it is more promising to use either cold atoms in optical lattices, where three-photon bound states have recently been observed [35], or arrays of superconducting qubits [36]. For atoms the interactions can be controlled optically using the Feshbach resonances [37], and for superconducting qubits just by applying external voltage [38].

\section{MODEL FOR SCATTERING AND DOUBLON STATES}

The structure under consideration is schematically illustrated in Fig. 1. It is described by the Hamiltonian

$$
\begin{aligned}
H= & \hbar \omega_{0} \sum_{j=1}^{N} a_{j}^{\dagger} a_{j}+J \sum_{j=1}^{N-1}\left(a_{j}^{\dagger} a_{j+1}+a_{j+1}^{\dagger} a_{j}\right) \\
& +U \sum_{j=1}^{N} \frac{\left[1-(-1)^{j}\right]}{2} a_{j}^{\dagger} a_{j}\left(a_{j}^{\dagger} a_{j}-1\right),
\end{aligned}
$$

where $a_{j}^{\dagger}\left(a_{j}\right)$ are the photon creation (annihilation) operators, $J$ is the tunneling parameter, and $U$ is the photon-photon interaction strength. The photon-photon interaction term $2 U$ is present only for every second cavity.

We look for the two-photon solutions of the Hamiltonian (1), where the wave function has the form

$$
|\Psi\rangle=\sum_{j, j^{\prime}=1}^{N} \Psi_{j j^{\prime}} a_{j}^{\dagger} a_{j^{\prime}}^{\dagger}|0\rangle,
$$

with $\Psi_{j j^{\prime}}=\Psi_{j^{\prime} j}$, reflecting the bosonic nature of the excitations. We substitute the wave function in the Schrödinger equation $E \Psi=H \Psi$ with the Hamiltonian (1) and obtain a system of linear equations for the coefficients $\Psi_{j j^{\prime}}$ in Eq. (2). As such, the interacting two-particle problem in one dimension is exactly mapped to the noninteracting single-particle problem in two dimensions.

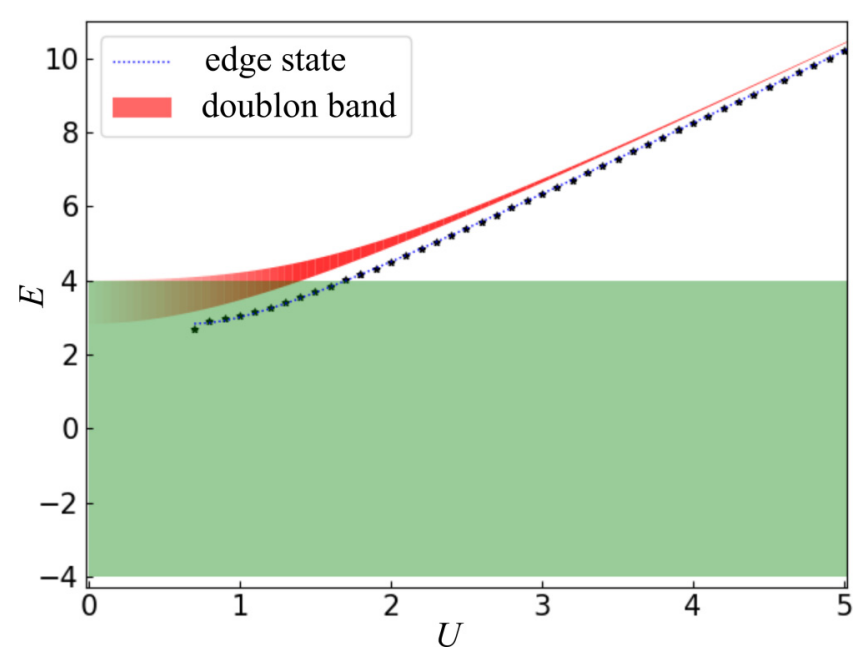

FIG. 2. Energy spectra of the photon pairs depending on the interaction strength $U$. Entire green rectangle is the scattering states continuum. Red branch is a manifold of doublon states, blue dashed line shows the energy of the edge state, calculated via Eq. (15), and the dotted line shows the numerically calculated energy of the edge state in a finite system with $N=30$ cavities. Calculation has been performed for the interaction strength $J=1$.

The energy spectra dependence on the interaction strength is shown in Fig. 2. From here on in order to simplify expressions, when speaking about energy, $E$, we will mean a value $E-2 \hbar \omega_{0}$ considering $2 \hbar \omega_{0}$ as zero energy level. The entire continuum between $E=-4 J$ and $E=4 J$, shown as green rectangle, is occupied by the scattering states, representing quasi-independent photons. For such states, the wave function is the tensor product of the wave functions of single photons and the energy is simply the sum of their energies, with a slight correction due to interaction term. Two branches emerge from this continuum. These are a band of bound photon pairs (red) that we will term as doublons from now on and the doublon edge state (blue), where both photons are localized at the same edge, respectively. In the finite system the edge state is clearly defined only in the case of strong interaction (see Sec. IV); therefore, in Fig. 2 relevant blue dashed line and dotted line are plotted up to $U=0.7 \mathrm{~J}$, which was found to be the lowest value, at which the edge state is preserved for $N=20$ cavities. The doublon band has a dispersion (8) derived below in Sec. III. The lower and higher edges of the doublon band correspond to $k= \pm \pi$ and $k=0$, respectively. The spatial distribution for these three basic types of two-photon states are illustrated in Fig. 3. Here, the two axes correspond to the coordinates of the two photons. The scattering state in Fig. 3(a) corresponds to quasi-independent photons without strong correlations in their coordinates. The doublon state in Fig. 3(b) is very different with large occupation probability at only half of the diagonal cells. This corresponds to the two photons that are spatially bound together by the spatially modulated interaction. Both Fig. 3(c) and Fig. 3(d) depict the edge state of the photon pair in logarithmic scale. In the case of strong interaction, Fig. 3(c), the exponential decay away from the corner shows the pure edge state, whereas for weak interaction in a finite system, the edge state energy $E \sim 2 U$ lies within the 
(a)

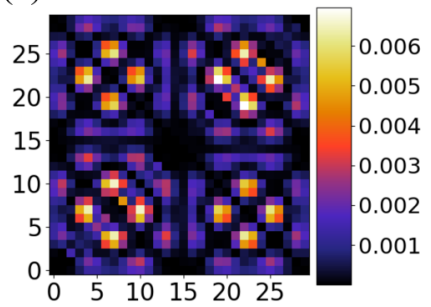

(c)

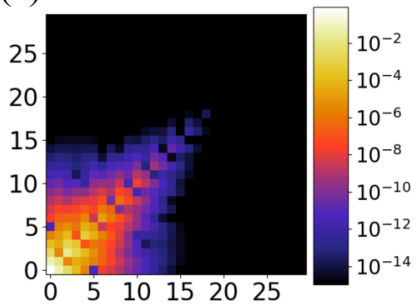

(b)

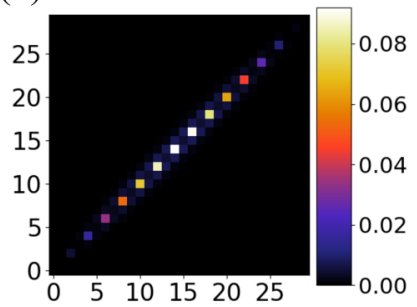

(d)

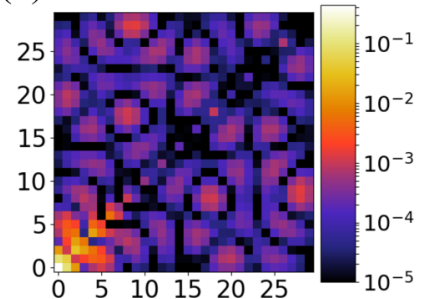

FIG. 3. Spatial distribution $\left|\Psi_{m n}\right|^{2}$ of four characteristic twophoton eigenmodes: (a) the scattering state, $E=3 \mathrm{~J}$; (b) doublon, $E=5.16 J$; (c) edge state, $E=4.52 J$; (d) edge state, $E=3.16 J$. The interaction strength is $U=2 J$ for the panels (a)-(c) and $U=$ $1.1 J$ for the panel $(\mathrm{d})$.

scattering continuum and, therefore, it is hybridized with scattering states. In this case, the decay is exponential only in the close proximity to the corner, and the state is partially delocalized; see Fig. 3(d).

Figures 2 and 3 present a basic overview of the eigenstates depending on the photon-photon interaction strength. Now we present a more detailed analysis of bulk doublon states (Sec. III) and edge states of doublons (Sec. IV).

\section{DOUBLON STATES}

In this section we derive analytically the doublon dispersion for the infinite system, and compare it with the numerical calculation for a finite array of cavities. The results for these two approaches match exactly. In order to find the bound photon states we use the Bethe ansatz like in [17,26], i.e., look for the wave function Eq. (2) in the form

$$
\Phi_{j j^{\prime}} \equiv\left(\begin{array}{c}
\Psi_{2 j, 2 j^{\prime}} \\
\Psi_{2 j, 2 j^{\prime}+1} \\
\Psi_{2 j+1,2 j^{\prime}+1} \\
\Psi_{2 j+1,2 j^{\prime}+2}
\end{array}\right)=\Phi e^{i k\left(j+j^{\prime}\right)+i q\left(j^{\prime}-j\right)},
$$

with $j<j^{\prime}$, where we write four values of the wave function in the unit cell as a one column vector. Here, $k$ is the center-of-mass wave vector and the wave vector $q$ describes the relative motion of the photons. Inserting this ansatz into the Hamiltonian Eq. (1) for $j \neq j^{\prime}$, i.e., in cavities outside of the main diagonal with red cells in Fig. 4, we obtain the dispersion of the scattering states:

$$
E=\left\{\begin{array}{l} 
\pm 4 J \cos \frac{q}{2} \cos \frac{k}{4}, \\
\pm 4 J \cos \frac{q}{2} \sin \frac{k}{4} .
\end{array}\right.
$$

Equation (4) indicates that two different wave vectors $q$ are possible for given eigenmode energy $E$ and the center-of-mass

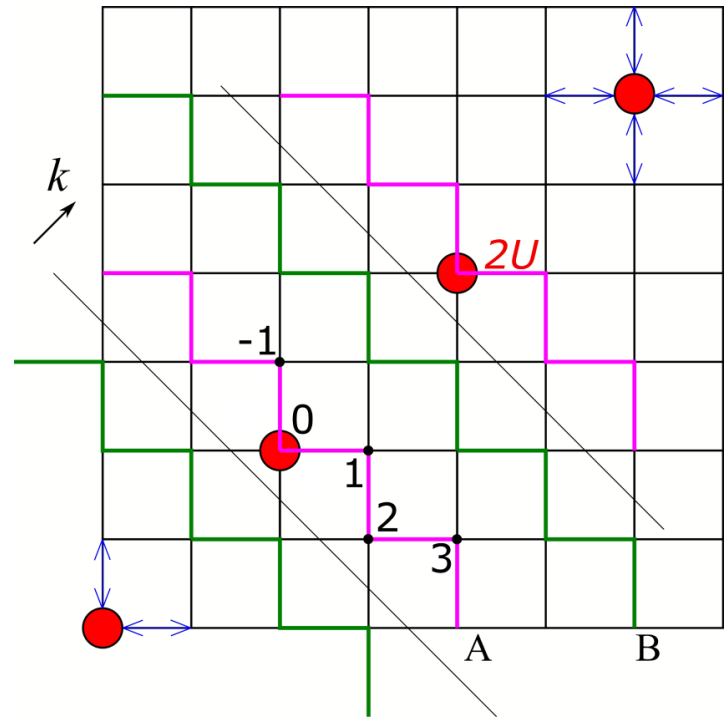

FIG. 4. Mapping of the one-dimensional two-particle problem onto the effective single-particle two-dimensional problem. Two coordinates of each node in the lattice correspond to the positions of the two photons; the links represent single-photon tunneling between the nearest sites. Red circles describe energy shift by $2 U$. Two gray lines bound the unit cell with the fixed center-of-mass coordinate of the photon pair; sites $A$ and $B$ are located on the pink and green lines, respectively.

wave vector $k$ :

$$
\cos \frac{q_{1}}{2}=\frac{E}{4 J \cos (k / 4)}, \quad \cos \frac{q_{2}}{2}=\frac{E}{4 J \sin (k / 4)} .
$$

Due to this degeneracy in $q$ we need to include a superposition of two waves with $q_{1}, q_{2}$ in the ansatz (3) in order to describe the edge state of doublons

$$
\Phi_{j j^{\prime}}=e^{\mathrm{i} k\left(j+j^{\prime}\right)}\left[\Phi_{1} e^{\mathrm{i} i q_{1}\left(j-j^{\prime}\right)}+\Phi_{2} e^{\mathrm{i} q_{2}\left(j-j^{\prime}\right)}\right],
$$

where

$$
\Phi_{1}=\left(\begin{array}{l}
a_{1} \\
b_{1} \\
c_{1} \\
d_{1}
\end{array}\right), \quad \Phi_{2}=\left(\begin{array}{l}
a_{2} \\
b_{2} \\
c_{2} \\
d_{2}
\end{array}\right) .
$$

Next, we examine the effect of the photon-photon interaction on the solution Eq. (6). Namely, we substitute (6) into the Hamiltonian (1) with $j=j^{\prime}$, and consider complex values of $q_{1}, q_{2}$ with the positive imaginary part, so that the wave function would decay with increase of the photon-photon distance. Rigorous solution results in the following implicit doublon dispersion law:

$$
\frac{1}{U}=\frac{1}{\sqrt{E^{2}-16 J^{2} \cos ^{2} \frac{k}{4}}}+\frac{1}{\sqrt{E^{2}-16 J^{2} \sin ^{2} \frac{k}{4}}} .
$$

Despite the fact that doublons are present in the spectrum for all $U>0$, the smaller the $U$, the smaller the photon-photon binding, as has been illustrated by the gradient shading of the doublon band in Fig. 2. This can be shown by rewriting Eq. (8) 
(a)

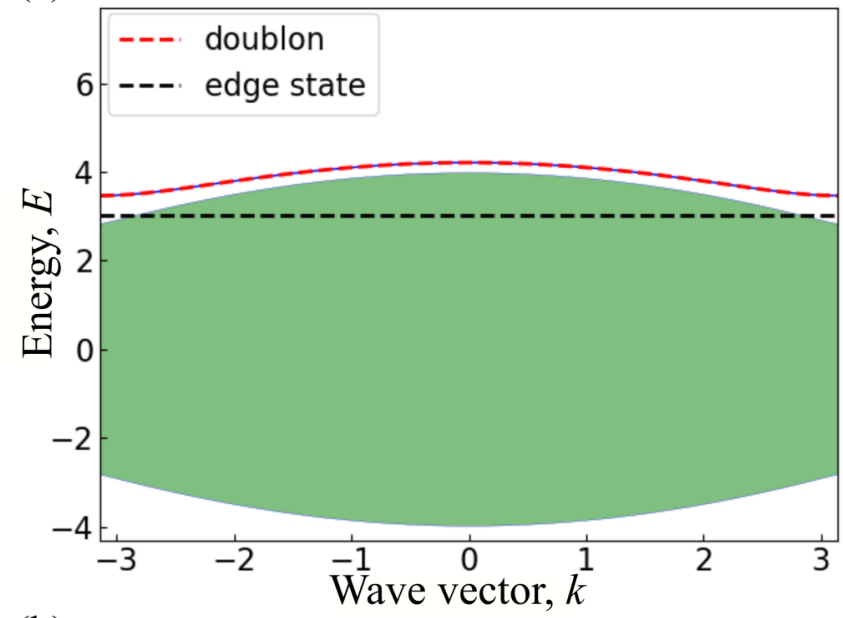

(b)

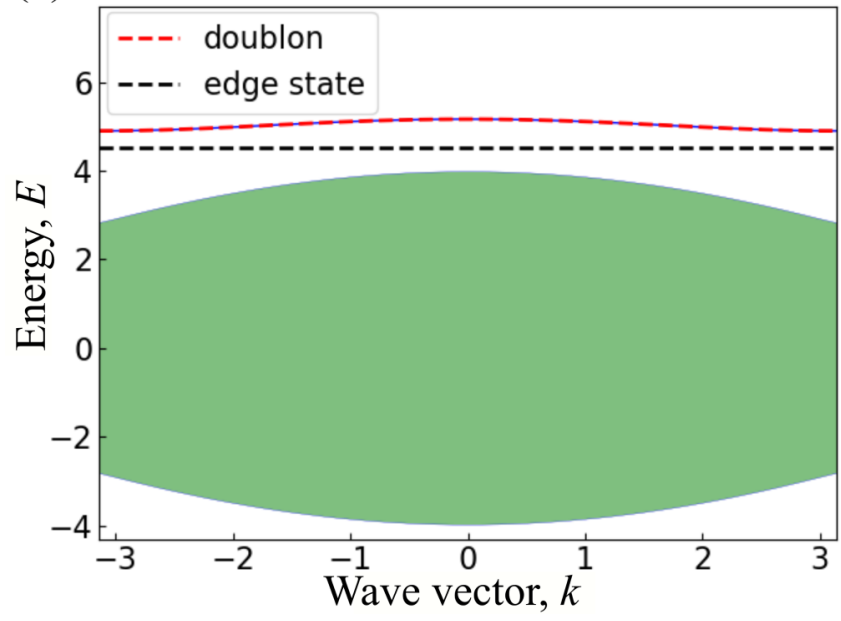

FIG. 5. Band structure of the photon pairs calculated for the interaction strength $U=1$ (a) and for $U=2$ (b). Red and black dashed lines show the doublon bands and edge states correspondingly, in both cases.

as

$$
\frac{E}{U}=\frac{1}{\tanh q_{1}^{\prime \prime}}+\frac{1}{\tanh q_{2}^{\prime \prime}},
$$

where $q_{1,2}^{\prime \prime}=\operatorname{Im}\left(q_{1,2}\right)$ are the inverse localization lengths. In the case of vanishing interaction, $U \rightarrow 0$, we find that either $q_{1}^{\prime \prime} \rightarrow 0$ or $q_{2}^{\prime \prime} \rightarrow 0$, which quenches the binding between the two photons. The two-photon band structure for two different regimes is illustrated in Fig. 5.

We will now proceed to obtain the band structure of doublons in the infinite lattice. To this end, we first fixed the center-of-mass wave vector $k$, using the ansatz (6), and then numerically solved the problem for a one-dimensional effective model describing relative motion of the two photons, shown in Fig. 4. The unit cell has a shape of a stripe (green and pink lines in Fig. 4) passing along the line of constant center of mass of two photons. The Hamiltonian, describing the relative photon motion, reads

$$
H=\left(\begin{array}{cc}
H_{A} & V \\
V^{\dagger} & H_{B}
\end{array}\right),
$$

where matrices $H_{A}, H_{B}, V$ are infinite and their central part is

$$
\begin{aligned}
H_{A} & =\left(\begin{array}{ccccc}
0 & J & 0 & 0 & 0 \\
J & 0 & J & 0 & 0 \\
0 & J & 2 U & J & 0 \\
0 & 0 & J & 0 & J \\
0 & 0 & 0 & J & 0
\end{array}\right), \\
H_{B} & =\left(\begin{array}{ccccc}
0 & J & 0 & 0 & 0 \\
J & 0 & J & 0 & 0 \\
0 & J & 0 & J & 0 \\
0 & 0 & J & 0 & J \\
0 & 0 & 0 & J & 0
\end{array}\right), \\
V & =\left(\begin{array}{ccccc}
0 & J e^{-i k} & 0 & 0 & 0 \\
J & 0 & J & 0 & 0 \\
0 & J e^{-i k} & 0 & J e^{-i k} & 0 \\
0 & 0 & J & 0 & J \\
0 & 0 & 0 & J e^{-i k} & 0
\end{array}\right) .
\end{aligned}
$$

Here, $H_{A}, H_{B}$ are Hamiltonians for the sites located at pink and green "stripes" in Fig. 4 correspondingly, and $V$ represents their interaction. Our calculations were performed for the matrices of the finite size $61 \times 61$, and the result is shown in Fig. 5. The green area and the red and black dashed lines represent scattering continuum and doublon band, calculated via (4) and (8), and the edge state, respectively. Blue lines are numerical eigenstates. For scattering states and doublon band numerical and analytical results perfectly agree. The doublon band Eq. (8) overlaps spectrally with the scattering states for $U<\sqrt{2} J$ and is spectrally separated for $U>\sqrt{2} J$. This is directly seen from the comparison of Figs. 5(a) and 5(b) and also agrees with the dependence on the interaction strength calculated in Fig. 2.

\section{EDGE STATE OF THE BOUND PHOTON PAIR}

The interaction-induced two-photon edge states in the system have been found numerically in Figs. 2 and 3. These edge states are not topologically protected in the usual sense. We were not able to relate them to the usual topological invariance such as Chern numbers or Zak phases, or to demonstrate their robustness to some specific kind of disorder. However, we still find the considered edge states quite special because, contrary to usual Tamm states [17], they arise in a periodic structure without any apparent defects at the edge. Even though the first cavity is nonlinearly detuned from the second one, the third one has the same energy as the first one. Hence it is not immediately obvious why the tunneling from the first cavity to the third one will not couple it to the bulk of the structure and delocalize all the excitations. In this section we consider the limit of strong interaction regime $(U \gg J)$, where the presence of edge states can be understood qualitatively by applying the perturbation theory. Visually, this can be illustrated in Fig. 4: the lattice nodes constitute the unperturbed Hamiltonian, red circles stand for the photon-photon interaction, and the tunneling constants $J$ (links between the nodes) represent a small perturbation.

We start with the separation of the Hamiltonian (1) into the unperturbed part $H_{0}$, determined only by the photon-photon interaction, and a perturbation $V$, including the tunneling 
effects:

$$
\begin{aligned}
H_{0}= & \sum_{j=1}^{N} \hbar \omega_{0} a_{j}^{\dagger} a_{j} \\
& +U \sum_{j=1}^{N} \frac{\left[1-(-1)^{j}\right]}{2} a_{j}^{\dagger} a_{j}\left(a_{j}^{\dagger} a_{j}-1\right), \\
\hat{V}= & J \sum_{j=1}^{N-1}\left(a_{j}^{\dagger} a_{j+1}+a_{j+1}^{\dagger} a_{j}\right) .
\end{aligned}
$$

From now on, we will consider the case of an odd number of cavities in the array $N$, so that both edge sites have a nonzero nonlinear interaction term and array has mirror symmetry. The eigenstates of Eq. (12) have then the $(N+1) / 2$ degeneracy with $E^{(0)}=2 U$. Since we focus only on these states, we will enumerate them in a new way by using only one index:

$$
\Psi_{n} \equiv \Psi_{2 n, 2 n}, \quad E_{n}^{(0)}=2 U, \quad 1 \leqslant n \leqslant \frac{N+1}{2} .
$$

The eigenenergies are enumerated correspondingly. This $(N+1) / 2$ degeneracy is lifted when the photon tunneling described by the operator $\hat{V}$ is taken into account. The photon tunneling can be visualized as quantum walks along the ribs of the graph in Fig. 4. The second-order processes include two ribs. Since the minimal distance between the nearest red cells in Fig. 4 is equal to four, the nearest red cells are not hybridized in the second order in $V$. On the other hand, there exist nonzero diagonal elements $V_{n n}^{(2)}$ that describe the shifts of the state energies induced by the tunneling (blue arrows in Fig. 4). These energy corrections are proportional to the number of nearest neighbors of the $n$th red cell, so the resulting energy correction is

$$
E_{n}^{(2)}= \begin{cases}J^{2} / U, & n=1 ;(N+1) / 2 \text { (edge state), } \\ 2 J^{2} / U, & 1<n<(N+1) / 2 \text { (doublons) }\end{cases}
$$

for the red cavities. Equation (15) is already sufficient to explain the formation of the edge states of the photon pairs. The energies of the edge sites are detuned from the energies of the bulk sites since these sites have less neighbors. As such, the hybridization of the edge sites with the bulk is suppressed and the localized edge states are formed. The blue line in Fig. 2 has been calculated according to Eq. (15) and well agrees with the result of numerical calculation of the edge state energy (black dots). Such formation mechanism is inherent for the few-particle quantum states in the one-dimensional lattices and can be understood as a quantum analog of the nonlinear self-trapping at the edge [26,31]. However, the distinctive ingredient of the considered model is the spatial modulation of the nonlinearity. If the nonlinearity were present both at the odd and even sites, the two-particle edge states would have not existed.

A similar approach can be extended to describe the formation of the bulk Bloch states of the doublons in the limit of strong interaction. This requires a consideration in the fourth order of perturbation theory. In this case the paths including four ribs should be included in Fig. 4 that can connect the neighboring red sites to each other. The secular equation has the form

$$
\sum_{n^{\prime}}\left(E^{(4)} \delta_{n n^{\prime}}-V_{n n^{\prime}}^{(4)}\right) c_{n^{\prime}}^{(0)}=0,
$$

where the indices $n, n^{\prime}$ run over the states that have remained degenerate after the second order correction has been taken into account, and $V_{n n^{\prime}}^{(4)}$ corresponds to a bulk expression including the terms, proportional to $\sum_{m, l, k} V_{n m} V_{m l} V_{l k} V_{k n^{\prime}} /(2 U)^{3}$. After the careful calculation of the matrix elements, we obtain the following system of equations for the sites $n$ :

$$
\begin{gathered}
\left(E-2 J^{2} / U\right) \Psi_{n}=\frac{J^{4}}{2 U^{3}}\left(\Psi_{n-1}+\Psi_{n+1}\right) \\
\times\left(1<n<\frac{N+1}{2}\right), \\
\left(E-J^{2} / U\right) \Psi_{1}=\frac{J^{4}}{2 U^{3}} \Psi_{2}, \\
\left(E-J^{2} / U\right) \Psi_{\frac{N+1}{2}}=\frac{J^{4}}{2 U^{3}} \Psi_{\frac{N-1}{2}} .
\end{gathered}
$$

The system of equations (18) has a transparent interpretation. It describes an effective one-dimensional tight binding model for doublon with the tunneling constant $t=J^{4} / 2 U^{3}$, and the energy detuning for the first and last cavities is equal to $\Delta E=$ $J^{2} / U$, leading to the formation of the edge states.

As was mentioned above, the presence of the edge state can be qualitatively explained by analyzing the quantum walks along the ribs of the graph in Fig. 4. In order to explain this idea in a simple way, we will introduce new notations. From now on, $|n m\rangle$ denotes a state, where one photon is in the $n$th site and another one is in the $m$ th site. Starting from the state $|n m\rangle$ and making a one tunneling step, it is possible to reach only the states $|n, m \pm 1\rangle$ and $|n \pm 1, m\rangle$, which is illustrated with ribs in Fig. 4. The transition $|11\rangle \rightarrow$ $|11\rangle$-from nonlinear corner state back to itself-goes in two steps $(|11\rangle \rightarrow|12\rangle \rightarrow|11\rangle)$, whereas the transition $|11\rangle \rightarrow$ $|33\rangle$ - from corner state to the other nearest nonlinear staterequires four steps. Since the second-order energy correction for the two-photon edge state $|11\rangle$ differs from those for other nonlinear states, $|2 n+1,2 n+1\rangle$, Eq. (15), the spectral separation of the edge state occurs before hybridization with the whole ensemble into doublons, which preserves it in the energy spectra.

It is instructive to generalize this analysis and directly compare our system with systems with differing number of photons and/or differing type of interaction modulation. We consider an array of cavities with the following Hamiltonian:

$$
\begin{aligned}
H_{m}= & \hbar \omega_{0} \sum_{j=1}^{N} a_{j}^{\dagger} a_{j}+J \sum_{j=1}^{N-1}\left(a_{j}^{\dagger} a_{j+1}+a_{j+1}^{\dagger} a_{j}\right) \\
& +U \sum_{j=1}^{N} f_{m j} a_{j}^{\dagger} a_{j}\left(a_{j}^{\dagger} a_{j}-1\right),
\end{aligned}
$$

where

$$
f_{m j}= \begin{cases}0, & \text { if } j-1 \bmod m \neq 0, \\ 1, & \text { if } j-1 \bmod m=0\end{cases}
$$


(a)

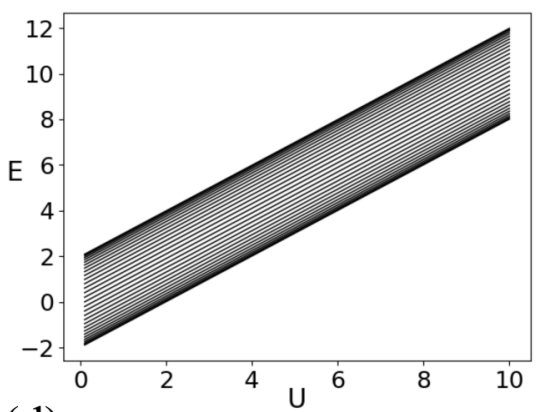

(d)

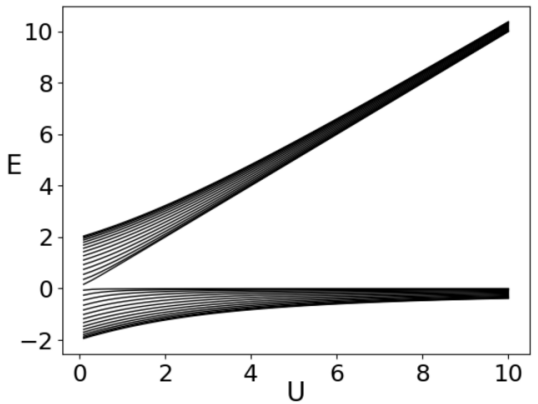

(g)

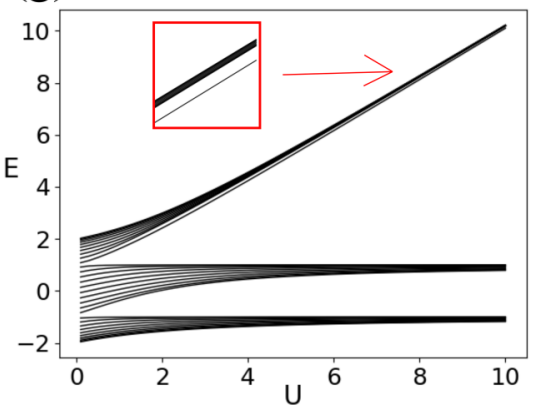

(b)

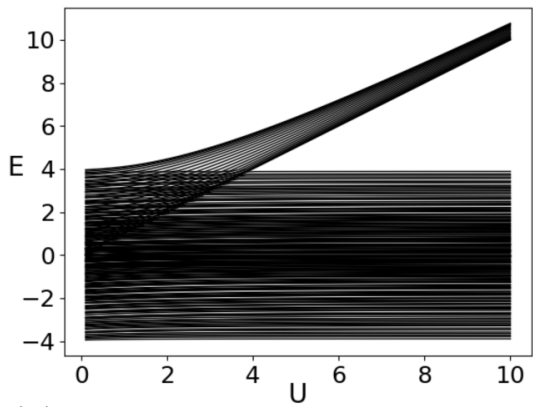

(e)

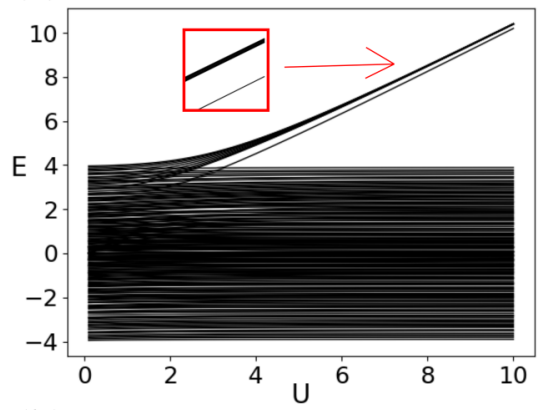

(h)

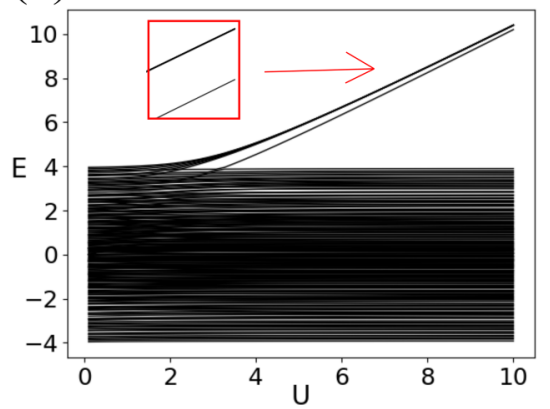

(c)

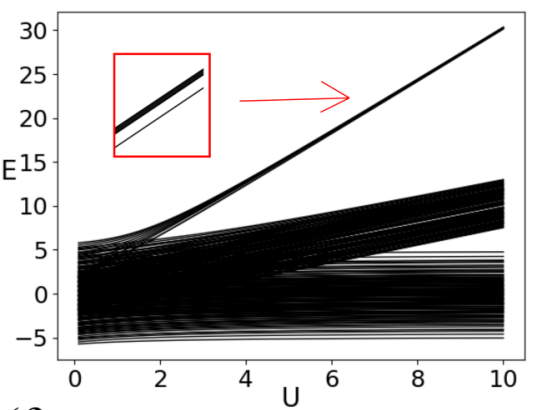

(f)

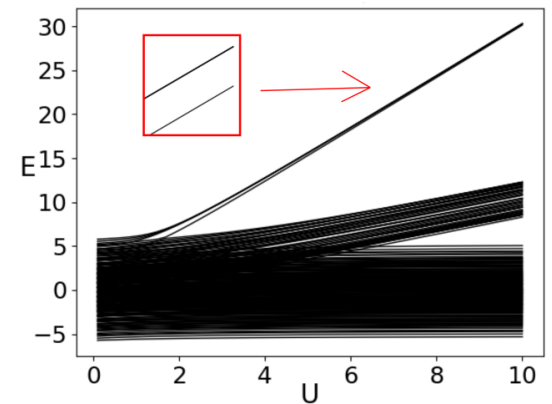

(i)

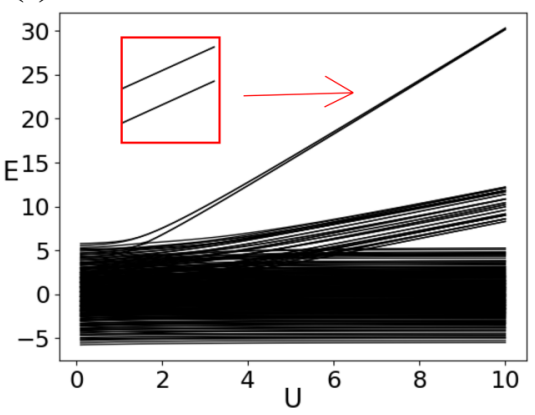

FIG. 6. Energy spectra dependence on interaction constant $U$ depending on the interaction modulation period and number of photons. First, second and third columns correspond to arrays with one photon and 30 cavities, two photons and 20 cavities, and three photons and 10 cavities, respectively. The three rows correspond to the nonlinear interaction on every site, every second, and third site, respectively. In all red insets the upper thick branch corresponds to the states with all photons in the nonlinear sites in the bulk and thin line below is the edge state with all photons in the edge site.

stands for the period of modulation of interaction. In the case $m=2$ the Hamiltonian (19) is exactly the Hamiltonian (1) for our problem with the modulation period 2. In the case of $m=$ 1 , i.e., without the modulation, the transition between nearest nonlinear sites, $|11\rangle \rightarrow|22\rangle$, requires just two steps, same as $|11\rangle \rightarrow|11\rangle$, and edge state should hybridize with other doublons. In any other case when $m \geqslant 2$, the transition between nearest nonlinear sites, $|11\rangle \rightarrow|m+1, m+1\rangle$, needs $2 m>2$ steps and the edge state emerges in the spectrum.

The analysis above demonstrates that the edge states arise due to the general features of two-photon propagation in the bulk, rather than the presence of the defect on the edge as usually happens for the Tamm states. This is conceptually similar to the topological edge states and makes it tempting to introduce global discrete indices characterizing the nontrivial two-photon propagation. In fact, similar consideration has already been performed in our earlier work [26] on a two-photon
Su-Schrieffer-Heeger model, where the tunneling constants have been modulated in space instead of the nonlinearity in the current work. We have linked the existence of the edge states to local vertex connectivity of the quantum walk graph being greater than 4 [26]. Our analysis can also be applied to higher numbers of photons inside the array. For $n=3$ photons, edge state, which is three-photon-edge state in this case, occurs even without the modulation, i.e., for $m=1$. The reason is that the transition $|111\rangle \rightarrow|111\rangle$ still takes two steps, but $|111\rangle \rightarrow|222\rangle$ is now three. With increasing number of photons this tendency persists, i.e., the edge state remains without modulation for any $n \geqslant 3$ number of photons in the array. Since the states with high photon numbers admit classical interpretation, the edge localization in the quantum problem for high photon numbers can be interpreted as linked to the nonlinear self-localization in the classical discrete nonlinear Schrödinger equation [31]. 
It is also instructive to rewrite the Hamiltonian (19) for the system with one photon in the form

$$
H_{m}=\sum_{j=1}^{N} a_{j}^{\dagger} a_{j}\left(\hbar \omega_{0}+f_{m j} U\right)+J \sum_{j=1}^{N-1}\left(a_{j}^{\dagger} a_{j+1}+a_{j+1}^{\dagger} a_{j}\right),
$$

which means detuning of on-site energy for every $m$ th site. The same reasoning shows that the edge state will occur only in $m \geqslant 3$ in this case.

The whole analysis of the two above paragraphs is confirmed by the results of direct numerical calculation presented in Fig. 6. Nine panels show the energy spectra of the systems with the number of photons and the period of modulation increasing from one to three. It was found numerically that six of them have edge states. These states correspond to the panels located in the lower-right corner of the figure, i.e., high photon numbers or modulation period, in full agreement with our analysis. The edge states are zoomed in the insets of the plots, shown by the red rectangles.

\section{SUMMARY}

To summarize, we have considered theoretically the manybody energy spectrum in the array of nonlinear cavities with spatially modulated photon-photon interaction. We have examined the dependence of the spectra on the number of photons and on the modulation period. In the specific case when the interaction is nonzero only for every second cavity, we have found the two-particle eigenstates of the infinite system analytically from the Bethe ansatz. They can be divided into the scattering states, where the two photons are quasi-independent from each other and the doublons, i.e., the two-photon states, bound by the interaction. We demonstrate that for sufficiently strong interaction the system features two-photon edge states, with both photons localized at the same edge of the array. The presence of such doublon edge states requires spatial modulation of the nonlinearity; they are absent if the interaction parameter is the same for all cavities. The formation of edge states has been interpreted in the regime of strong interaction as a nonlinear self-localization at the edge. We have revealed a universal behavior of the states, depending on the interaction modulation period and the number of excitations, that both tend to promote the selflocalization. These results were understood analytically from the structure of the graph of many-body quantum walks. Our results might be useful to understand the energy spectrum and the mechanisms of edge state formation in various quantum systems, from structured polaritonic cavities to the arrays of superconducting qubits.

\section{ACKNOWLEDGMENTS}

We thank M. A. Gorlach and N. A. Olekhno for useful discussions. This work has been supported by the Russian Foundation for Basic Research Grants No. 18-29-20037 and No. 18-32-20065. A.P. has been partially supported by the Presidium of RAS (Program 13, Nanostructures in Life Sciences).
[1] H. Bernien, S. Schwartz, A. Keesling, H. Levine, A. Omran, H. Pichler, S. Choi, A. S. Zibrov, M. Endres, M. Greiner, V. Vuletić, and M. D. Lukin, Probing many-body dynamics on a 51-atom quantum simulator, Nature (London) 551, 579 (2017).

[2] A. Keesling, A. Omran, H. Levine, H. Bernien, H. Pichler, S. Choi, R. Samajdar, S. Schwartz, P. Silvi, S. Sachdev, P. Zoller, M. Endres, M. Greiner, V. Vuletić, and M. D. Lukin, Quantum Kibble-Zurek mechanism and critical dynamics on a programmable Rydberg simulator, Nature (London) 568, 207 (2019).

[3] I. Carusotto and C. Ciuti, Quantum fluids of light, Rev. Mod. Phys. 85, 299 (2013).

[4] A. Peruzzo, M. Lobino, J. C. F. Matthews, N. Matsuda, A. Politi, K. Poulios, X.-Q. Zhou, Y. Lahini, N. Ismail, K. Worhoff, Y. Bromberg, Y. Silberberg, M. G. Thompson, and J. L. OBrien, Quantum walks of correlated photons, Science 329, 1500 (2010).

[5] A. S. Solntsev, F. Setzpfandt, A. S. Clark, C. W. Wu, M. J. Collins, C. Xiong, A. Schreiber, F. Katzschmann, F. Eilenberger, R. Schiek, W. Sohler, A. Mitchell, C. Silberhorn, B. J. Eggleton, T. Pertsch, A. A. Sukhorukov, D. N. Neshev, and Y. S. Kivshar, Generation of Nonclassical Biphoton States Through Cascaded Quantum Walks on a Nonlinear Chip, Phys. Rev. X 4, 031007 (2014).

[6] S. Mittal, E. A. Goldschmidt, and M. Hafezi, A topological source of quantum light, Nature (London) 561, 502 (2018).
[7] S. Barik, A. Karasahin, C. Flower, T. Cai, H. Miyake, W. DeGottardi, M. Hafezi, and E. Waks, A topological quantum optics interface, Science 359, 666 (2018).

[8] A. Blanco-Redondo, B. Bell, D. Oren, B. J. Eggleton, and M. Segev, Topological protection of biphoton states, Science 362, 568 (2018).

[9] P. Roushan, C. Neill, A. Megrant, Y. Chen, R. Babbush, R. Barends, B. Campbell, Z. Chen, B. Chiaro, A. Dunsworth, A. Fowler, E. Jeffrey, J. Kelly, E. Lucero, J. Mutus, P. J. J. O’Malley, M. Neeley, C. Quintana, D. Sank, A. Vainsencher, J. Wenner, T. White, E. Kapit, H. Neven, and J. Martinis, Chiral ground-state currents of interacting photons in a synthetic magnetic field, Nat. Phys. 13, 146 (2016).

[10] D. C. Mattis, The few-body problem on a lattice, Rev. Mod. Phys. 58, 361 (1986).

[11] K. Winkler, G. Thalhammer, F. Lang, R. Grimm, J. H. Denschlag, A. J. Daley, A. Kantian, H. P. Büchler, and P. Zoller, Repulsively bound atom pairs in an optical lattice, Nature (London) 441, 853 (2006).

[12] P. M. Preiss, R. Ma, M. E. Tai, A. Lukin, M. Rispoli, P. Zupancic, Y. Lahini, R. Islam, and M. Greiner, Strongly correlated quantum walks in optical lattices, Science 347, 1229 (2015).

[13] A. Schreiber, A. Gábris, P. P. Rohde, K. Laiho, M. Štefaňák, V. Potoček, C. Hamilton, I. Jex, and C. Silberhorn, A 2d quantum walk simulation of two-particle dynamics, Science 336, 55 (2012). 
[14] G. Corrielli, A. Crespi, G. Della Valle, S. Longhi, and R. Osellame, Fractional Bloch oscillations in photonic lattices, Nat. Commun. 4, 1555 (2013).

[15] N. A. Olekhno, E. I. Kretov, A. A. Stepanenko, D. S. Filonov, V. V. Yaroshenko, B. Cappello, L. Matekovits, and M. A. Gorlach, Topological edge states of interacting photon pairs realized in a topolectrical circuit, arXiv:1907.01016.

[16] M. Lyubarov and A. Poddubny, Exceptional points for photon pairs bound by nonlinear dissipation in cavity arrays, Opt. Lett. 43, 5917 (2018).

[17] S. Longhi and G. D. Valle, Tamm-Hubbard surface states in the continuum, J. Phys.: Condens. Matter 25, 235601 (2013).

[18] L. Lu, J. D. Joannopoulos, and M. Soljačić, Topological states in photonic systems, Nat. Phys. 12, 626 (2016).

[19] T. Ozawa, H. M. Price, A. Amo, N. Goldman, M. Hafezi, L. Lu, M. C. Rechtsman, D. Schuster, J. Simon, O. Zilberberg, and I. Carusotto, Topological photonics, Rev. Mod. Phys. 91, 015006 (2019).

[20] Z. Wang, Y. Chong, J. D. Joannopoulos, and M. Soljacic, Observation of unidirectional backscattering-immune topological electromagnetic states, Nature (London) 461, 772 (2009).

[21] P. St-Jean, V. Goblot, E. Galopin, A. Lemaître, T. Ozawa, L. Le Gratiet, I. Sagnes, J. Bloch, and A. Amo, Lasing in topological edge states of a one-dimensional lattice, Nat. Photonics 11, 651 (2017).

[22] S. Kruk, A. Poddubny, D. Smirnova, L. Wang, A. Slobozhanyuk, A. Shorokhov, I. Kravchenko, B. Luther-Davies, and Y. Kivshar, Nonlinear light generation in topological nanostructures, Nat. Nanotechnol. 14, 126 (2019).

[23] J.-L. Tambasco, G. Corrielli, R. J. Chapman, A. Crespi, O. Zilberberg, R. Osellame, and A. Peruzzo, Quantum interference of topological states of light, Sci. Adv. 4, eaat3187 (2018).

[24] G. Salerno, M. Di Liberto, C. Menotti, and I. Carusotto, Topological two-body bound states in the interacting Haldane model, Phys. Rev. A 97, 013637 (2018).

[25] M. Di Liberto, A. Recati, I. Carusotto, and C. Menotti, Twobody physics in the Su-Schrieffer-Heeger model, Phys. Rev. A 94, 062704 (2016).

[26] M. A. Gorlach and A. N. Poddubny, Topological edge states of bound photon pairs, Phys. Rev. A 95, 053866 (2017).

[27] M. Di Liberto, A. Recati, I. Carusotto, and C. Menotti, Twobody bound and edge states in the extended SSH Bose-Hubbard model, Eur. Phys. J.: Spec. Top. 226, 2751 (2017).
[28] M. A. Gorlach, M. Di Liberto, A. Recati, I. Carusotto, A. N. Poddubny, and C. Menotti, Simulation of two-boson bound states using arrays of driven-dissipative coupled linear optical resonators, Phys. Rev. A 98, 063625 (2018).

[29] B. A. Bernevig and T. L. Hughes, Topological Insulators and Topological Superconductors (Princeton University Press, Princeton, NJ, 2013).

[30] M. A. Gorlach and A. N. Poddubny, Interaction-induced twophoton edge states in an extended Hubbard model realized in a cavity array, Phys. Rev. A 95, 033831 (2017).

[31] R. Pinto, M. Haque, and S. Flach, Edge-localized states in quantum one-dimensional lattices, Phys. Rev. A 79, 052118 (2009).

[32] A. Kavokin, J. J. Baumberg, G. Malpuech, and F. P. Laussy, Microcavities (Clarendon Press, Oxford, 2006).

[33] G. Muñoz-Matutano, A. Wood, M. Johnsson, X. Vidal, B. Q. Baragiola, A. Reinhard, A. Lemaître, J. Bloch, A. Amo, G. Nogues, B. Besga, M. Richard, and T. Volz, Emergence of quantum correlations from interacting fibre-cavity polaritons, Nat. Mater. 18, 213 (2019).

[34] Á. Cuevas, J. C. L. Carreño, B. Silva, M. De Giorgi, D. G. Suárez-Forero, C. S. Muñoz, A. Fieramosca, F. Cardano, L. Marrucci, V. Tasco, G. Biasiol, E. del Valle, L. Dominici, D. Ballarini, G. Gigli, P. Mataloni, F. P. Laussy, F. Sciarrino, and D. Sanvitto, First observation of the quantized exciton-polariton field and effect of interactions on a single polariton, Sci. Adv. 4, eaao6814 (2018).

[35] Q.-Y. Liang, A. V. Venkatramani, S. H. Cantu, T. L. Nicholson, M. J. Gullans, A. V. Gorshkov, J. D. Thompson, C. Chin, M. D. Lukin, and V. Vuletić, Observation of three-photon bound states in a quantum nonlinear medium, Science 359, 783 (2018)

[36] Z. Yan, Y.-R. Zhang, M. Gong, Y. Wu, Y. Zheng, S. Li, C. Wang, F. Liang, J. Lin, Y. Xu, C. Guo, L. Sun, C.-Z. Peng, K. Xia, H. Deng, H. Rong, J. Q. You, F. Nori, H. Fan, X. Zhu, and J.-W. Pan, Strongly correlated quantum walks with a 12-qubit superconducting processor, Science 364, 753 (2019).

[37] C. Chin, R. Grimm, P. Julienne, and E. Tiesinga, Feshbach resonances in ultracold gases, Rev. Mod. Phys. 82, 1225 (2010).

[38] J. Koch, T. M. Yu, J. Gambetta, A. A. Houck, D. I. Schuster, J. Majer, A. Blais, M. H. Devoret, S. M. Girvin, and R. J. Schoelkopf, Charge-insensitive qubit design derived from the Cooper pair box, Phys. Rev. A 76, 042319 (2007). 